\title{
An Adaptive Mobile Phone Bandwidth Allocation System for Disaster and Emergency Communications
}

\author{
Onur Cagirici and Mehmet S. Unluturk
}

\begin{abstract}
Since the population density grows in metropolis, more and more people lose their lives in case of disasters. In order to locate and save these people, communication tools are essential. Therefore, the importance of communication is increased recently. Systems such as Global Positioning System (GPS) are not consistent enough to locate and save a person on time. We have developed a system that allows cellular phone users to communicate directly, in a very short time. We use frequency-division multiplexer (FDM) together with time-division multiplexer (TDM) to give all the users enough time to communicate. In this architecture, each person who desires to make phone calls in case of an emergency would be able to establish calls with a round-robin algorithm. The system is also able to adjust the time limit for the users.
\end{abstract}

Index Terms-Emergency communications, bandwidth allocation, adaptive systems.

\section{INTRODUCTION}

Communication becomes highly crucial when there is an emergency situation e.g. earthquake. However, there are a number of reasons that make people unable to communicate with each other. In these cases, the lack of communication causes many life losses [1]. Moreover, investigations show that half of the deaths occur within the first two hours of the disaster [2]. The most common way to be informed about the status of a person is cellular phones. Especially, when an earthquake strikes, there are several major problems about mobile communications [3]. Unfortunately, for each operator, there is a limit for simultaneous communications. Hence, several methods were developed to avoid lack of communications. In 2002, Meissner et al. pointed out the difficulties of designing a system for disaster communication in The First IEEE Workshop on Disaster Recovery Networks [4]. In the same conference, Midkiff et al. presented a novel system for disaster and emergency response [5]. Those presentations contained the information that there are great challenges for developing an adaptive system. In 2007, Rao et al. investigated the methods for using IT in emergencies [6] In 2008, a system that uses GSM backhauling is developed in order to allocate more bandwidth for the area of disaster [7]. In 2011, Duressi et al. developed "an adaptive architecture for ad hoc communications to be used in emergency conditions" [8]. All approaches so far are concerned about either manipulating the bandwidth of the cell-phones, or using ad hoc mode to determine the location of another person. Furthermore, GPS is erroneous about 50m (164ft)

Manuscript received January 22, 2013; revised March 24, 2013.

The authors are with the University of Economics, Dept. of Software Engineering 35330 Izmir, Turkey (e-mail: onur.cagirici@ieu.edu.tr, suleyman.unluturk@ieu.edu.tr). horizontally and about $100 \mathrm{~m}(328 \mathrm{ft})$ vertically [9]. In our approach, we set time limitations for each user, which allow a huge number of users to communicate when used with the approaches described in this paper. Notice that, the best location information can be provided by a person. If the person is alive, (s)he may describe if (s)he is effected by the disaster. If so, a person's description would be more precise and powerful than any system. It is obvious that in case of emergency, more than usual number of people try to make phone calls to their friends and relatives. Since the cell phone networks use time-division multiplexing (TDM), when the limit is reached, no more users are able to make phone calls. However, in case of a disaster, intuitively, a person who is able to make phone calls, would not desire to end the call. This situation is the main reason for lack of communication. If an operator has the capacity of serving at most $U$ number of users, another user will receive the server busy tone. Our system avoids unnecessarily long communications using frequency-division multiplexer (FDM) together with TDM.

\section{SYSTEM ARCHITECTURE}

Cell phone communications are continuous. FDM technique is used for mobile phone communications. In emergency situations, such as earthquake, the main issue is congestion. Since there is no time limit for users, when the total frequency bandwidth capacity is reached, no more users are able to communicate. In some cases, communication may be essential. However, when there is a scarcity, users do not want to end their communication. Since the cell phone operators do not use Time-Division Multiplexing, most of the users are not able to communicate in an emergency. In our paper, we design a system that is adaptive for congestion using both FDM and TDM, allowing all the users to communicate with each other in a short time.

Consider that the total bandwidth of a mobile phone operator is B and each user needs A amount of bandwidth allocated in order to make phone calls. That means the operator is able to serve at most $B / A$ number of phone calls at a time. Consider that an average user uses bandwidth for $\mathrm{T}$ seconds. That is, with full capacity, seconds are needed for a group of users to finish their calls on average. Consider that there are U numbers of user pairs, who desire to make a call, it takes seconds for all the users to communicate. That is, usually, in case of emergencies, more than usual number of users desire to make calls. Here, we use TDM to limit the time that two users communicate.

$$
\begin{gathered}
\frac{B}{A} \times T \\
\frac{U}{A} \times T=\frac{U \times \frac{A}{B} \times T=\frac{U A T}{B}}{}
\end{gathered}
$$


There is a protocol that prevents a user to continue talking in each operator. If one uses credits, he/she cannot keep talking on the phone when their credit is over. If one uses billed tariff, the operator blocks the calls when the due date for bills is reached. Our purpose is to use this protocol in order to prevent over-usage of an allocated bandwidth in emergency situations.

Consider that every user has L seconds to finish the call. If they do not end the communication, the operator will cut the line. As a result $\mathrm{T}$ is replaced by $\mathrm{L}$ in the equation. Hence, the equation becomes

$$
\frac{U A L}{B}
$$

For sake of simplicity let us say that

$$
\frac{U A T}{B}=C_{u}
$$

and

$$
\frac{U A L}{B}=C_{l}
$$

Notice that $C_{u}$ denotes unlimited calls and $C_{l}$ denotes limited calls. Hence, by using (3) and (4), we can say that

$$
\frac{C_{u}}{C_{l}}=\frac{T}{L}
$$

In (3), UAL/B seconds are enough for all users will be able to inform each other. To be more precise, consider that an operator has 60 million users and at most 500 thousand user pairs can communicate with each other simultaneously. That is,

$$
\frac{B}{A}=5 \times 10^{5}
$$

Consider that each phone call continues 100 seconds in average.

$$
T=10^{2}
$$

Even if one of every six user desire to make phone calls(that is 5 million pairs). That is, seconds to serve all the users. That is 1000 seconds, approximately 16.6 minutes. Also, notice that there is no queuing system in cell phone operators. Hence, if any user fails to make a call, he/she should continuously try until a pair of user ends their phone call. This brings up energy problems (batteries last shorter when a call is attempted) besides the communication problems [11], a system for time limitations would not be sufficient to solve both problems. A user still can run out of battery trying to establish a call.

$$
U=5 \times 10^{6}
$$

Thus, by (3), it takes

$$
\frac{10^{2} \times\left(5 \times 10^{6}\right)}{5 \times 10^{5}}
$$

In order to prevent the situations described above, we use a queuing system besides time limitations. First, consider that we use 10 seconds of time limit, that is $\mathrm{L}=10$. Hence, $\mathrm{C}_{\mathrm{u}} / \mathrm{C}_{\mathrm{l}}=10$, by (6), when considering the example case described above. That means it takes 200 seconds for all users, who desire to make calls, to communicate. Consider that all of the users of the operator try to make phone calls (60 million users, 30 million pairs), with 10 seconds of time division, by (3), it takes 600 seconds, that is 10 minutes. With a simple queuing system, users will be informed about the waiting time and will not continuously try to establish a call.

\section{A. Multiplexing Algorithm}

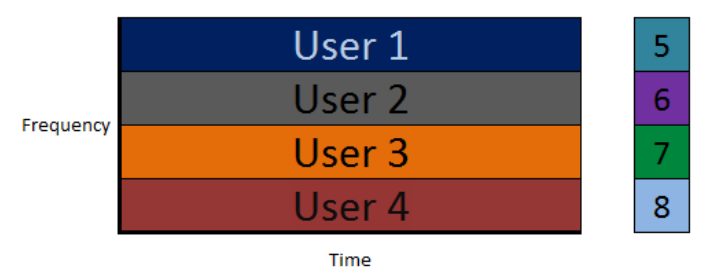

Fig. 1. FDM.

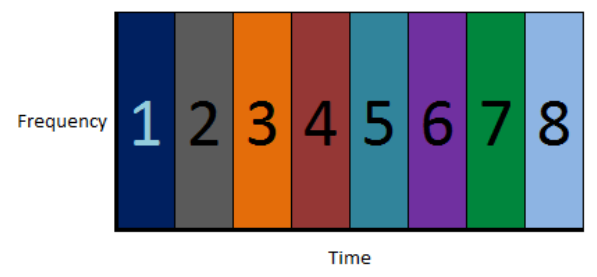

Fig. 2. TDM.

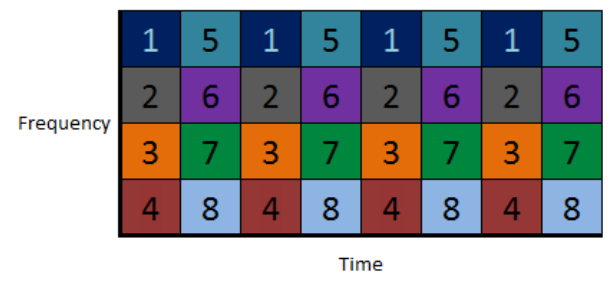

Fig. 3. FDM with TDM.

In Fig. 1, we show the current multiplexing of cell phone operators. There are four users who are able to make phone calls (Users 1 to 4). Besides, there are four more users who are hearing the operator busy tone (Users 5 to 8 ). There is only one way for any of the waiting users to establish a connection. That is, at least one of the four communicating users ends their phone call. Fig. 2 represents TDM where users have a time limit. When we use TDM, within the same period, all users are able to communicate with each other. Fig. 3 shows our system, which uses both multiplexing techniques, and also a queuing algorithm. Within the same period, all the users are able to communicate four times.

\section{B. Queuing Algorithm}

The queuing system steps in when the capacity of the operator is full and more users try to establish phone calls. Notice that, some mobile numbers are defined as emergency numbers and those numbers will have no time limits or more time limits than the ordinary users.

while the capacity is not full

do

establish connection

end while

while any user $U$ tries to make a call

do

add $\mathrm{U}$ to queue

end while

while queue is not empty

do

serve the next user

end while 


\section{Call Ending Algorithm}

Meanwhile, there is another algorithm that runs during the phone calls and ends the call when the time limit is reached. When a user is dropped, the system automatically appends that user to the queue. Thus, the system prevents continuous call attempts. The call will be automatically established when there is an empty slot.

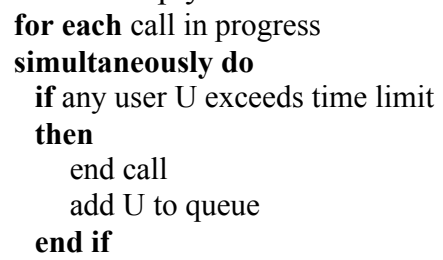

\section{Time Slot Determination}

It is obvious that, after people are informed about their relatives, there is less number of users who want to communicate. Therefore, people who need more time to get informed, or have not been informed yet, might use more time after a while. The system does not allow unnecessarily long phone calls, but if the number of users is small, it would be redundant to have a very short time limit, as can be seen in Fig. 3. In this case, both the system and the users are unnecessarily busy. In this case, we have a number of options.

The first solution is to remove the time limit. That option is applied if the average for a user to end a call is less than the time limit. That is, the value of formula (6) is less than 1.

The second solution is to extend the time limit. When we set a value $\alpha$, and the value of (6) is between 1 and $\alpha$, we apply the second solution.

\section{CONCLUSIONS}

We have developed an adaptive system that goes online in case of disasters and emergencies. The system manipulates multiplexing strategy of cellular phone operators. The system is also enterable. It does not prevent localization techniques developed before. Moreover, it makes people available to describe their location, which is more accurate than any other localization technique. Setting a reasonable time limit helps people to get informed about each other and prevents unnecessarily long phone calls, hence the congestion and service unavailability. Using a queuing algorithm would prevent continuous call attempts. Moreover, dynamically changing the time limit would prevent unnecessary number of disconnections between users.

\section{REFERENCES}

[1] D. R. Griffin, "The 9/11 Commission Report," The National Commission on Terrorist Attacks Upon the United States, 2004.
[2] ITU, "ITU Corporate Annual Report 2006," ITU/ESCAP Regional Workshop on Disaster Communications, Bangkok, Thailand, December, 2006.

[3] A. Vervacek. Mobile communications and earthquakes: a very "disturbing" marriage. [Online]. Available: http://earthquake-report.com/2011/09/16/mobile-communications-and -earthquakes-a-very-disturbing-marriage/

[4] R. R. Rao, J. Eisenberg, and T. Schmitt, "Improving disaster management: the role of IT in mitigation, preparedness, response, and recovery," National Research Council, Washington D.C., 2007.

[5] E. H. Fazli, M. Werner, N. Courville, M. Berioli, and V. Boussemart, "Integrated GSM/WiFi backhauling over satellite: flexible solution for emergency communications," IEEE Vehicular Technology Conference (VTC), 2008.

[6] H. Kunreuther and M. Useem, Learning from catastrophes. Strategies for reaction and response, Wharton School Publishing, New Jersey, 2009.

[7] A. Durresi, M. Durresi, V. Paruchuri, and L. Barolli, "Ad Hoc communications for emergency conditions," Advanced Information Networking and Applications (AINA), IEEE International, 2011.

[8] A. Meissner, T. Luckenbach, T. Risse, T. Kirste, and H. Kirchner, "Design challenges for an integrated disaster management communication and information system," The First IEEE Workshop on Disaster Recovery Networks (DIREN), 2002.

[9] S. F. Midkiff and C. W. Bostian, "Rapidly-Deployable broadband wireless networks for disaster and emergency response," The First IEEE Workshop on Disaster Recovery Networks (DIREN), 2002.

[10] M. S. Grewal, L. R. Weill, and A. P. Andrews, Global positioning systems, inertial navigation, and integration, USA: John Wiley \& Sons, 2004.

[11] A. Rahmati and L. Zhong, "Human-battery interaction on mobile phones," Pervasive and Mobile Computing, 2009.

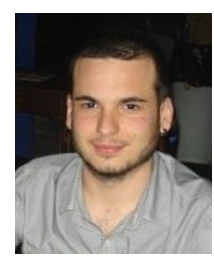

Onur Cagirici was born in Izmir, on March 31, 1989. Finished B.Sc in software engineering, Izmir University of Economics (IEU), Izmir, in 2011. Still is an employee and master's degree student of IEU. Interested in combinatorial optimization, computational geometry and aglorithm design.

In year 2010, he earned Erasmus scholarship and went to Universidad de Cantabria, Spain for internship. In September 2011, he started his master's degree in International Computer Institute (ICI), Agean University, Izmir. In November 2011, he started working as research assistant in IEU, Izmir. In February 2012, he began doing his master's degree in IEU with full scholarship. In September 2012, he began working on a project that is being supported and funded by TUBITAK, "The Scientific and Technological Research Council of Turkey" and conducted by Assist. Prof. Dr. Huseyin Akcan.

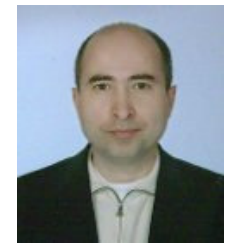

Mehmet S. Unluturk was born in Zonguldak, on April 22, 1967. Finished Electronics and Telecommunications Engineering in Istanbul Technical University. He got a scholarship and got his Masters of Science and PhD degrees in the field of Electrical and Computer Engineering from Illinois Institute of Technology, Chicago, USA.

He worked for Panasonic (Chicago) and General Electric (Chicago) for 11 years. He has expertise in the field of nurse call systems, HL7, Wireless Phones, RFID, Pocket Pagers, Real Time Location Systems, Whiteboard, and Hospital Staff Assignments. In his free time, he applies the neural network techniques to the Organic-Conventional Food Classification, Bio-crystallization, and detection of antibiotics in milk products. He has three US patents in the field of nurse call systems. 